\title{
Cascaded techniques for improving emphysema classification in computed tomography images
}

\author{
Musibau A. Ibrahim, Ramakrishnan Mukundan \\ Department of Computer Science and Software Engineering, University of Canterbury, Christchurch, New Zealand
}

Received: May 21, 2015

DOI: $10.5430 /$ air.v4n2p112
Accepted: July 7, 2015

URL: http://dx.doi.org/10.5430/air.v4n2p112

\begin{abstract}
The previous studies demonstrated the effectiveness of the multi-fractal based method for the classification of histo-pathological cases by calculating the local singularity coefficients of an image using different intensity measures. This paper proposed to improve the previous results by investigating the features derived from the combination of the alpha-histograms and the multifractal descriptors in the classification of Emphysema in computed tomography (CT) images. The performances of the classifiers are measured by using the classification accuracy (error matrix) and the area under the receiver operating characteristic curve (AUC). And further, the experimental results compared well with the local binary patterns (LBP) approach, a state-of-the-art measure for pulmonary Emphysema. The results also show that the proposed cascaded approach significantly improves the overall classification accuracy.
\end{abstract}

Key Words: Emphysema classification, Multi-fractal analysis, Multi-fractal spectrum, Histogram comparison, Statistical self-similarity

\section{Introduction}

Emphysema is one of the main components of chronic obstructive pulmonary diseases (COPD); it is characterized by loss of lung tissue and may eventually lead to gradual destruction of the lung. Detection and classification of Emphysema are therefore very important as this may lead to improved computer-aided diagnosis (CAD) ${ }^{[1]}$ Diagnosing Emphysema usually requires pulmonary function tests (PFTs), combined with a history of symptoms. The main tool through which the tests are performed is the spirometer; however, PFTs are not capable of detecting COPD at early stages. Another popular tool for diagnosing Emphysema is high resolution computed tomography (HRCT) imaging. CT imaging is a suitable method for demonstrating the presence, distribution and extent of Emphysema pat- terns in images. ${ }^{[1]}$ Emphysema in HRCT is characterized by the presence of areas of abnormally low attenuation, which can be easily contrasted with surrounding normal lung parenchyma. ${ }^{[2]}$ Emphysema can be classified into three different classes: centrilobular Emphysema (CLE), paraseptal Emphysema (PSE) and panlobular Emphysema (PLE). Sorensen has recently introduced new methods for texture analysis in CT images using local binary patterns (LBP) that achieved promising results in the classification of Emphysema subtypes.

The intensity distribution of lung tissue images is highly irregular and does not often permit a direct definition of shape parameters using geometrical descriptors. One approach towards extracting relevant features is to make use of the statistical self-similarities in local intensity variations. Most biomedical images exhibit such statistical self-similarity, a

*Correspondence: Musibau A. Ibrahim; Email: mai35@uclive.ac.nz; Address: Department of Computer Science and Software Engineering, University of Canterbury, Christchurch, New Zealand. 
repetition of form over a variety of scales. Several methods of multi-fractal analysis of medical images have been suggested and evaluated in different ways. ${ }^{[3-8]}$ Hemsley and Mukundan $^{[3]}$ developed a two-pass algorithm for the computation of multi-fractal spectrum and used the calculated spectra for the classification in a tissue image database. In Ref., ${ }^{[4]}$ the Holder exponent for the power law approximation of intensity measures in pixel neighbourhood is used for resolving local density variations in the CT lung images.

Multi-fractal analysis could find applications in Emphysema classification in CT images of the lung, but not much research has so far been done in this area. Recently in Ref., ${ }^{[9]}$ we had proposed a classification algorithm using multi-fractal descriptors. This paper provides an improved method using a combination of two different descriptors (multi-fractal spectra and alpha-histograms) for obtaining higher accuracy in the results. The first approach combines the features obtained from the multi-fractal descriptors and the alpha-histograms in the form of hybrid for the classification process. The second idea uses the area under the ROC curves to identify the best features that could yield maximum classification accuracy and high computational efficiency. The remainder of this paper is organized as follows: In section 2, different intensity measures used for the computation of Holder exponent and the overview of the multifractal methods are outlined. Experimental results and discussion are given in section 3 . Finally, section 4 summarizes the work and outlines some future directions.

\section{Material and methods}

The online CT Emphysema database ${ }^{[10]}$ used for this research consists of 168 non-overlapping annotated ROIs of size $61 \times 61$ pixel patches from three different classes: NT (59 never-smokers), CLE (50 healthy smokers), and PSE (59 smokers with COPD). ${ }^{[11]}$ The system overview for the multi-fractal approach of Emphysema classification is shown in Figure 1. The process involves several algorithmic stages, first of which is the calculation of the Holder exponent ( $\alpha$-values) at each pixel using a pre-selected intensity measure defined in pixel neighbourhoods. This computation is explained in detail in Section 2.1. The $\alpha$-values describe the variation in the local density of the image with respect to the chosen measure. The collection of all $\alpha$-values for a given image is referred to as the $\alpha$-image. The range of $\alpha$-values is subdivided into a number of small intervals, effectively decomposing the $\alpha$-image into several disjoint image slices. Each $\alpha$-image slice represents the collection of pixels in the input image having similar intensity variation (obeying similar power-law relationship in the intensity measure) across the pixel neighbourhoods. The traditional box-counting method is used for the calculation of the fractal dimension $f(\alpha)$ of the $\alpha$-images, providing the multifractal spectrum. The pixels having similar $\alpha$-values collectively yield a $\alpha$-histogram. Both the $\alpha$-histogram and the

Published by Sciedu Press multi-fractal spectrum contain highly discriminating texture features. Such features are gathered to form a descriptor and used in the image classifier.

\subsection{Holder exponent and multi-fractal measures}

The multi-fractal analysis describes the fractal properties of an image using an intensity-based measure within the neighbourhoods of each pixel. The local singularity coefficient, also known as the Holder exponent ${ }^{[5,12-14]}$ reveals the local behaviour of a measure function denoted as $\mu_{p}(w)$ where $w$ stands for the window size centred at the pixel $p$ (see Figure 2 ). The variation of the intensity measure with respect to $w$ can be characterized as follows:

$$
\begin{aligned}
& \mu_{p}(w)=C w^{\alpha_{p}} \\
& w=2 k+1, k=0,1,2, \cdots, m
\end{aligned}
$$

where $C$ is an arbitrary constant, and $m$ is the total number of boxes used in the computation of $\alpha_{p}$. The value of $\alpha_{p}$ can be estimated from the slope of the linear regression line in a $\log$-log plot where $\log \left(\mu_{p}(w)\right)$ is plotted against $\log (w)$. Some commonly used multi-fractal intensity measures used for the computation of the Holder exponent are the summation measure, the iso-surface measure, the maximum intensity measure and the inverse minimum measure. The final multi-fractal spectrum computed from an image obviously depends on the choice of the intensity-based measure that represents local variations of intensity values.

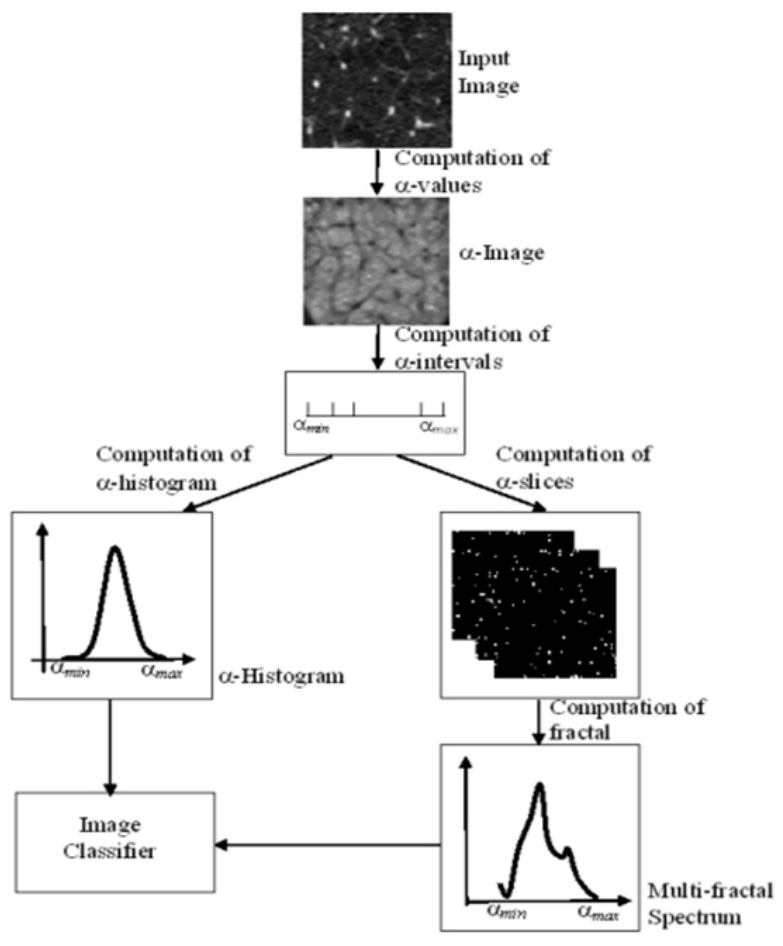

Figure 1: System overview of multi-fractal based classification of CT 


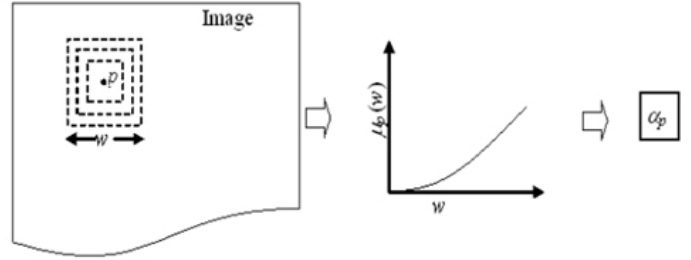

Figure 2: The Holder exponent is computed across multiple windows

A brief description of the intensity measures is given below. The sum measure $\mu_{p}^{[\text {sum }](w)}$ is defined as the sum of the intensity values within a local neighbourhood $w$. The measure could be further normalized using the total mass intensity; the normalized measure prevents values from becoming exceedingly large, which may lead to numerical instability. ${ }^{[3,9,15]}$

$$
\mu_{p}^{[\text {sum }]}(w)=\sum_{q \in w} I_{q}
$$

where $I_{q}$ denotes the intensity value at a pixel $q$ within the window $w$. The iso measure $\mu_{p}^{[i s o]}(w)$ counts the number of pixels that have the same intensity value with the centred pixel $p$ in a neighbourhood. If the centred pixel is the only pixel with unique intensity in the region, then measure has a value 1 .

$$
\mu_{p}^{[i s o]}(w)=\left\{q I_{q} \cong I_{p} ; q \in w\right\}
$$

where \# is the number of pixels. To account for minor variations in intensity values and noise, a threshold is often used in the comparison in (4). The inverse-minimum measure is $\mu_{p}^{[m i n]}(w)$ defined as follows:

$$
\mu_{p}^{[m i n]}(w)=1-\min _{q \in w} I_{q}
$$

In (5), the intensity values are assumed to be normalized in the range [0 1], so that the value resulting from the subtraction is always positive. The subtraction from 1 is used to meet the requirement that the measure does not decrease in value with increasing window size. The maximum measure is the measure with the greatest intensity found in the window $w$ centred at the pixel $p$.

$$
\mu_{p}^{[\max ]}(w)=\max _{q \in w} I_{q}
$$

\section{$2.2 \alpha$-Images and the multi-fractal spectrum}

The $\alpha_{p}$ values at pixels $p$ obtained from the previous step define a range $\left[\alpha_{\min }, \alpha_{\max }\right]$ of the real line, which is further divided into $n$ discrete steps $\alpha_{1}, \alpha_{2}, \alpha_{3}, \cdots, \alpha_{n}$. Each intermediate value $\alpha_{k}$ is defined as follows:

$$
\begin{aligned}
& \alpha_{k}=\alpha_{\min }+(k-1) \alpha_{k}, k=1,2, \cdots, n \\
& \Delta \alpha_{k}=\left(\alpha_{\max }-\alpha_{\min }\right) / n
\end{aligned}
$$

For our experimental analysis, we used the value $n=100$. The following images and histogram values are readily obtained:

$\alpha$-image:

$$
I_{p}^{(\alpha)}=\left(\frac{\alpha_{p}-\alpha_{\min }}{\alpha_{\max }-\alpha_{\min }}\right) 255
$$

$\alpha$-histogram:

$$
h_{k}=\#\left\{p \mid \alpha_{p} \in\left[a_{k}, a_{k+1}\right]\right\}, k=1,2, \cdots, n-1
$$

$\alpha$-slices:

$$
A_{k}=\left\{p \mid \alpha_{p} \in\left[a_{k}, a_{k+1}\right]\right\}, k=1,2, \cdots, n-1
$$

An $\alpha$-slice $A_{k}$ is a binary image the only foreground pixels (with maximum intensity value) are those pixels that satisfy the condition in (11). It therefore represents the collection of pixels in the original image where the values of the chosen intensity measure show the same power law variation.

\subsection{Multi-fractal spectrum}

An important texture feature that could be used in classification is the statistical self-similarity property exhibited by the sub-images represented by the $\alpha$-slices. Each $\alpha$-slice can be characterised by its fractal dimension. We subdivide an $\alpha$-slice $A_{k}$ into a regular grid of boxes with integral box sizes $\varepsilon$. The boxes containing at least one foreground pixel as given in (11) are counted, giving the number $N_{k}(\varepsilon)$. The box sizes are recursively scaled by half, as per the well-known box-counting algorithm, ${ }^{[14]}$ and the fractal dimension of the $\alpha$-slice estimated as the slope of the linear regression line on the $\log -\log$ plot with $\log (\varepsilon)$ along the $x$-axis, and $\log \left(N_{k}(\varepsilon)\right)$ along the $y$-axis. We denote this fractal dimension by $f\left(\alpha_{k}\right)$. The variation of $f\left(\alpha_{k}\right)$ with $\alpha_{k}, k=1,2, \cdots, n-1$ gives the multi-fractal spectrum.

\section{Experimental results and discussion}

In this section, we outline experimental results obtained using images from the Emphysema database, ${ }^{[10]}$ based on the implementation of the methods discussed in the previous sections. The feature vectors extracted from the calculated multi-fractal spectra and the alpha-histograms are used for classification and retrieving purposes. The results of the multi-fractal descriptors obtained for the three classes of the Emphysema images using four multi-fractal intensity measures (Section 2.1) are presented in Figure 3.

The histogram descriptors used for the classification experiments are constructed by dividing the range of $\alpha$-values generated from the Holder exponent into 100 intervals. The alpha-histogram is calculated for each alpha bin as the number of pixel counts with the $\alpha$-values within the $\alpha$-range $\left[\alpha_{i} \alpha_{i+1}\right]$. The average of the alpha-histogram for four randomly selected images is calculated and the feature vectors obtained from the descriptors are trained with the classifier 
algorithm. The alpha-histograms for each Emphysema class using the summation measure for the computation of Holder exponent are given in Figure 4.

The first stage of the classification uses the summation intensity measures for the Emphysema classification with different distance metrics. ${ }^{[15]}$ The summary of the classification results is shown in Table 1.

Table 1: Classification results using the summation measures

\begin{tabular}{lllll}
\hline & Predicted & & & \\
\hline \multirow{4}{*}{ Actual } & & NT & CLE & PSE \\
& NT & 100 & 0 & 0 \\
& CLE & 50 & 50 & 0 \\
& PSE & 17 & 25 & 55 \\
\hline
\end{tabular}

It can be observed from the results in Table 1 that there is a clear separation between the normal Emphysema class and other pathological cases, but some CLE classes are misclassified as the normal (NT) classes while some PSE classes also give some classification errors. In the second stage of the classification, the normal Emphysema class is removed such that the alpha-histogram descriptor is used for further classification of the two pathological classes (CLE and PSE). The Naïve Bayes classifier is introduced to this process, we applied the holdout partition method to divide the observations into training sets and test sets. ${ }^{[16]}$ There is a scalar specifying the proportion of the number of observations to be randomly selected for validation. In order to achieve promising results since the accuracy of the classifiers majorly depends on the training data; we consider 70 percent of the feature vectors for the training and 30 percent for testing. The performance of the classifiers is evaluated in the form of confusion matrix. A confusion matrix can be represented as a matrix $M \in R^{k \times k}$, a square matrix whose diagonal elements represents the actual classification accuracy where $k$ is the number of classes in the dataset. The classification error of the classifiers can be calculated as follows:
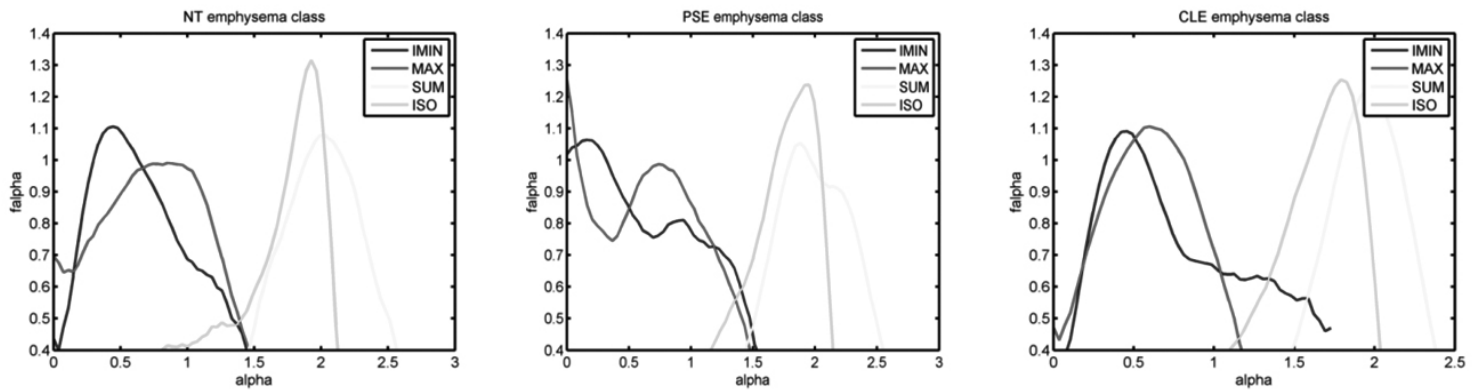

Figure 3: Multi-fractal descriptors for three classes of Emphysema images using each intensity measure
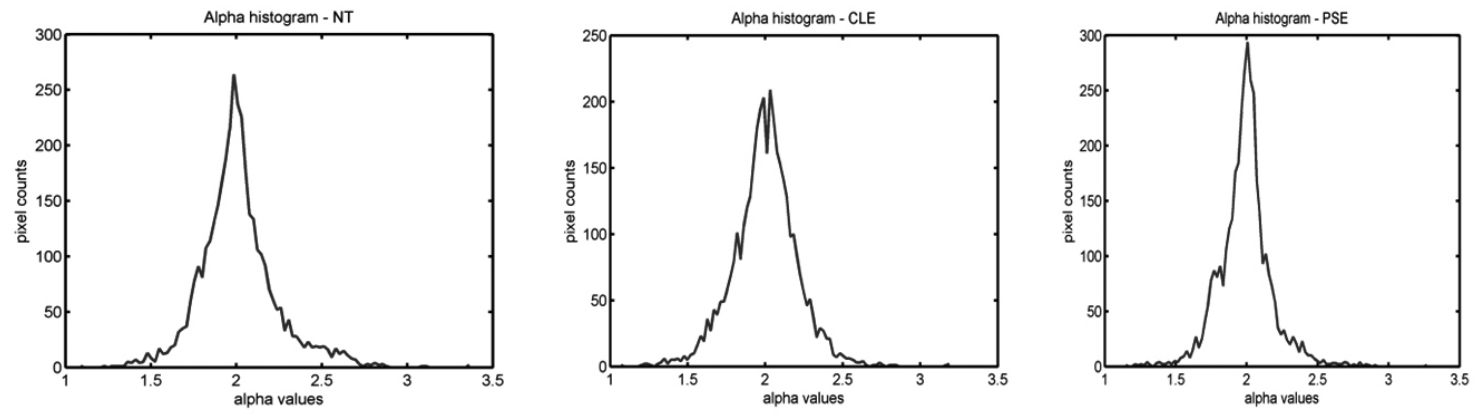

Figure 4: Alpha-histograms of each class of Emphysema image using the summation measure

$$
\text { Error }=1-\frac{\operatorname{trace}(\text { confusionMatrix })}{\text { sum(confusionMatrix })}
$$

where trace (.) is the sum of all the elements in the diagonal, and sum (.) is the sum of all the entries in the confusion matrix. The classification results obtained using the Naïve Bayes classifier over the alpha-histograms datasets are presented in Table 2. The entries in the diagonal cells of the tables presented are the percentage of the instances that are correctly classified, where the actual class matches the predicted classes, the entries off the diagonal are the percentage of the cases that are wrongly classified.

The results presented in Table 2 show good performance but with few errors. The next stage of the experiment would be to cascade this current result with the previous results obtained from the multi-fractal descriptors. The outlines of 
the experimental procedures are given in Figure 5.

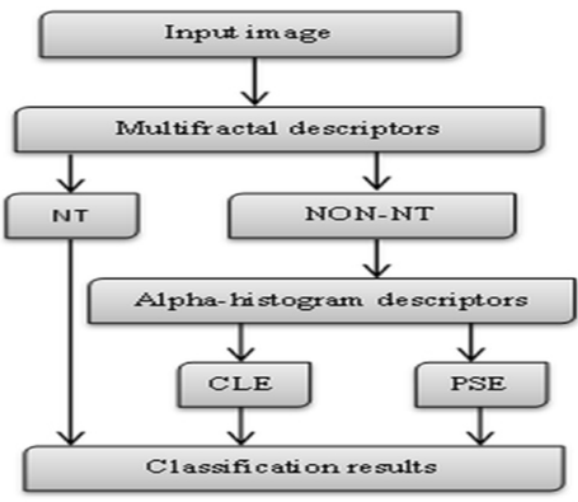

Figure 5: Outline of the experimental procedures for classification

It is noted from the results in Table 2 that the combination of information from the local singularity of the image (the alpha-histograms), combined with the information extracted from the multi-fractal descriptors, that is, the global features would form a powerful discriminating feature for the Emphysema classification. That is the reason for combining the results from the first stage of the classification to the second stage of the classification, and the final classification outputs are presented in Table 3.

The best two approaches investigated in Ref. ${ }^{[11]}$ are the LBP with an estimated classification accuracy of $95.2 \%$, and the Gaussian filter bank (GFB) histogram with an estimated accuracy of $94 \%$. The overall classification accuracy of $94.7 \%$ achieved by the proposed approach compared well with the two best approaches. The LBP result is slightly better, but gives few errors in separating the normal class from other pathological cases, $7 \%$ of the normal class are misclassified as PSE class. Our results clearly separated the normal class from the pathological cases (CLE and PSE), and outperformed the results achieved by the GFB approach. However, the results of the cascaded method indicate that the CLE and PSE classes have a higher number of misclassification errors than the GFB_LBP.

Table 2: Classification results for the two pathological classes

\begin{tabular}{llll}
\hline \multicolumn{4}{c}{ Predicted } \\
& Alpha-histograms descriptors \\
\hline \multirow{3}{*}{ Actual } & & CLE & PSE \\
& CLE & 92 & 8 \\
& PSE & 8 & 92 \\
\hline
\end{tabular}

Table 3: Classification results after combining the results from the two stages

\begin{tabular}{|c|c|c|c|c|}
\hline & $\begin{array}{l}\text { Pred } \\
\text { Fina }\end{array}$ & ation & & \\
\hline & & NT & CLE & PSE \\
\hline Actuol & NT & 100 & 0 & 0 \\
\hline Actud & CLE & $*$ & 92 & 8 \\
\hline & PSE & * & 8 & 92 \\
\hline
\end{tabular}

The results in Table 3 are achieved by direct combination of the results from the multi-fractal data sets in Table 1 and the classification results generated by the alpha-histogram descriptors in Table 2. The two cells in the Table are marked with the asterisk symbol, indicating there are no results between the pathological cases (CLE and PSE) and the normal Emphysema class (NT), since it has been eliminated from the data sets. The results achieved by this proposal show excellent performance with the overall classification accuracy of $94.7 \%$ (see Table 3). The final results are then compared with the two best approaches used in the recently published paper $^{[11]}$ as presented in Table 4.

Table 4: Comparison of classification results using cascaded approach and LBP

\begin{tabular}{|c|c|c|c|c|c|c|c|c|c|c|}
\hline & \multicolumn{10}{|c|}{ Predicted } \\
\hline & \multicolumn{5}{|l|}{ GFB } & \multicolumn{3}{|l|}{ LBP } & \multicolumn{2}{|c|}{ Our results } \\
\hline & & NT & CLE & PSE & NT & CLE & PSE & NT & CLE & PSE \\
\hline \multirow[t]{3}{*}{ Actual } & NT & 93 & 0 & 7 & 93 & 0 & 7 & 100 & 0 & 0 \\
\hline & CLE & 4 & 96 & 0 & 2 & 98 & 0 & * & 92 & 8 \\
\hline & PSE & 7 & 0 & 93 & 3 & 2 & 95 & * & 8 & 92 \\
\hline
\end{tabular}

The performances of the descriptors obtained from the alpha-histograms and the multi-fractal are also evaluated using the weighted area under the ROC curves. The area under the ROC curve is calculated for all possible combinations of the Emphysema class labels over the data sets derived from the hybrid combination of the alpha-histogram and the multi-fractal descriptors by using the Wilcoxon rank sum test. The average of the AUC for the possible class pair is calculated for each column and the column with the maxi- mum AUC's value is chosen as the best feature (see Table 5). Figure 6 presented the ROC curves for the selected features of the data sets and the corresponding class comparison of the AUC are given in Table 5.

Table 5: Pairwise AUCs for Emphysema Classes

\begin{tabular}{lllll}
\hline Class comparison & $\begin{array}{l}\text { NT vs. } \\
\text { CLE }\end{array}$ & $\begin{array}{l}\text { NT vs. } \\
\text { PSE }\end{array}$ & $\begin{array}{l}\text { CLE vs. } \\
\text { PSE }\end{array}$ & $\begin{array}{l}\text { Mean } \\
\text { AUC }\end{array}$ \\
\hline Best Features & 0.9204 & 0.7915 & 0.7633 & 0.8251 \\
\hline
\end{tabular}


Table 6: AUCs for Normal class $v s$. other cases and CLE vs. PSE

\begin{tabular}{llll}
\hline $\begin{array}{l}\text { Class } \\
\text { comparison }\end{array}$ & $\begin{array}{l}\text { NT vs. others } \\
\text { (ours) }\end{array}$ & $\begin{array}{l}\text { CLE vs. } \\
\text { PSE }\end{array}$ & $\begin{array}{l}\text { NT vs. others } \\
\text { (KNN) }\end{array}$ \\
\hline AUCs & 0.8559 & 0.7633 & 0.713 \\
\hline
\end{tabular}

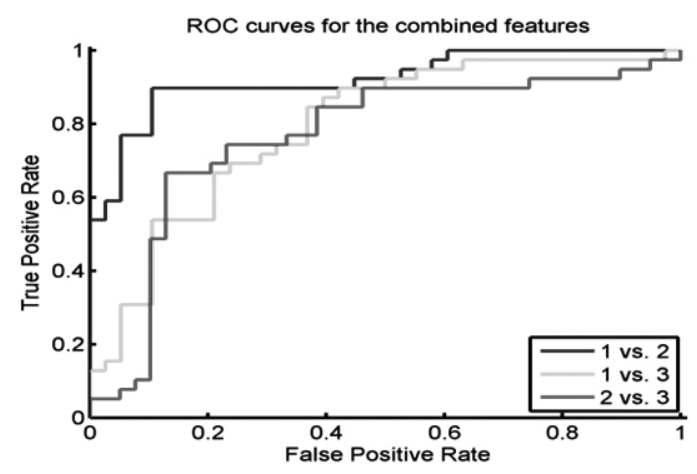

Figure 6: ROC curves of the combined features from the alpha-histograms and the Multi-fractal datasets using the best features

The Table 6 is generated from the pairwise class comparison results presented in Table 5 by adding the AUCs of the NT versus CLE and the NT versus PSE classes to produce NT versus other cases as indicated in Table 6. The closer the AUC's values to 1 , the better the performance of the data sets. It can be noted from the outcomes in Table 6 that the performances of the NT class versus other classes (CLE \& PSE) are better than the CLE class versus PSE class using the selected best features. This is also demonstrated in Figure 6 , the ROC curves of the NT class versus the CLE class are more separated than the ROC curve for the CLE class versus PSE class. The mean AUC of 0.8251, obtained by averaging all of the pairwise values in Table 5 is used for the selection of the best features. The result in Table 6 is then compared with another recent paper by Sorensen and Nielson. ${ }^{[17]}$ In Ref., ${ }^{[17]}$ the region of interest (ROI) classification for discriminating the Emphysema with and without COPD is calculated using the $k$ nearest neighbour approach and the AUC of 0.713 is achieved. In comparison with our results, the AUC value of 0.8559 obtained is significantly better than the 0.713 achieved by Sorensen. ${ }^{[17]}$ The major contributions of this study from the medical doctor's point of view is that the combined descriptor constructed after the cascaded approach, has significantly improved the overall classification accuracy of Emphysema images. The AUC value of 0.8559 also demonstrates the discriminating power of our approach in terms of separating the Emphysema with and without diseases; this is a great achievement since the separations between the normal and non-normal image samples are very important to the medical doctor or the radiologist.

\section{Conclusion}

This paper has presented a cascaded approach using the combined features of the multi-fractal and alpha-histograms descriptors for the classification of Emphysema in CT pulmonary images. The multi-fractal based descriptors demonstrate good performance in discriminating the normal Emphysema class from other pathological cases. Furthermore, the introduction of the alpha-histograms further improves the classification results. The performances of the descriptors are measured by the overall classification accuracy and the area under the ROC curves. Our results compared favourably well with the state-of-the-art measure for Emphysema, with an AUC of 0.8559 compared to 0.713. The proposed ideas significantly increase the classification accuracy, and confirm the effectiveness and robustness of the multi-fractal techniques in the classification of Emphysema images. The problem with the combined features is that the model complexity increases, which might slow the computational time and also increase the memory usage. The performance of the combined descriptors can be improved by parallelizing the classifier algorithms using the GPU parallel computing as this might improve the computational efficiency. Further effort would be to cascade the LBP-based approach with the multi-fractal techniques, and the results of the combined descriptors would be examined on the classification of Emphysema in CT images. The performances of the descriptors may be evaluated against other classification approaches.

\section{Acknowledgements}

The authors would like to thank Dr. Lauge Sorensen and other researchers for making available the online computed tomography Emphysema database used in this research.

\section{References}

[1] Chabat DHF, Yang G. Obstructive lung diseases: texture classification for differentiation at CT Radiology. 2003; 228(3): 871-877.

[2] Sørensen L, de Bruijne M. Dissimilarity representations in lung parenchyma classification, In Proceeding of the Spie digital library Conference, Medical imaging; Computer-Aided Diagnosis. 2009;
7260(27): 1-12. http://spie.org/Publications/Proceedi ngs/Paper/10.1117/12.812505

[3] Hemsley A, Mukundan R. Multifractal Measures for Tissue Image Classification and Retrieval; 11th IEEE Int. Symp. Multimed. 2009: 618-623. http://dx.doi .org10.1109/ISM. 2009.94

[4] Tay C, Mukundan R, Racoceanu D. Multifractal Analysis of Histopathological Tissue Images, In IVCNZ. 2011: 80-85. http: 
//hdl.handle.net/10092/6247

[5] Nilsson E. Multifractal-based Image Analysis with applications in Medical Imaging. Dep. Comput. Sci. Umea Univ. Umea Sweden. 2007: 33-70. www.cs.umu.se/education/examina/Rapporte r/EthelNilsson.pdf

[6] Stojić T, Reljin I, Reljin B. Adaptation of multifractal analysis to segmentation of microcalcifications in digital mammograms. Phys. A Stat. Mech. its Appl. 2006; 367: 494-508. www.sciencedirec t.com/science/article/pii/S0378437105012331

[7] Gavrovska A, Zajić G, Reljin I, et al. Classification of prolapsed mitral valve versus healthy heart from phonocardiograms by multifractal analysis. Comput. Math. Methods Med. 2013. http://dx.d oi.org/10.1155/2013/376152

[8] Vasiljevic J, Reljin B, Sopta J, et al. Application of multifractal analysis on microscopic images in the classification of metastatic bone disease. Biomed. Microdevices. 2012; 14: 541-548. www.ncbi.n Im.nih.gov/pubmed/22327812

[9] Ibrahim M, Mukundan R. Multi-fractal Techniques for Emphysema Classification in Lung Tissue Images. Int. Proc. Chem. Environ. Eng. 2014; 78: 115-119. http://ipcbee.com/list-106-1.h tml

[10] Computed Tomography Emphysema Database web portal [Internet]. Available from: http://image.diku.dk/emphysema_data base/. [Accessed:09-Feb-2015
[11] Sørensen L, Shaker SB, De Bruijne M. Quantitative Analysis of Pulmonary Emphysema using Local Binary Patterns. IEEE Trans. Med. Imaging. 2010; 29(2): 559-569. http://dx.doi.org/10.1109 /TMI . 2009. 2038575

[12] Vehel JL, Mignot P. Multifractal Segmentation of Images, Fractals, Image (Rochester, N.Y.). 1964; 2: 371-378. https://hal.inri a.fr/inria-00592260

[13] Mukundan R, Hemsley A. Tissue Image Classification Using Multi-Fractal Spectra, Int. J. Multimed. Data Eng. Manag. 2010; 1(2): 62-75. http://dx.doi.org/10.4018/978-1-4666-179 1-9. ch006

[14] Falconer K. Fractal Geometry-Mathematical Foundations and Applications., 2nd ed. London: Wiley. 2003. http://dx.doi.org/1 $0.2298 /$ A000204283R

[15] Choi E, Lee C. Feature extraction based on the Bhattacharyya distance. J. Pattern Recognit. Soc. 2003; 36: 1703-9. Available from: http://dx.doi.org/10.1016/S0031-3203(03)00035-9

[16] Zhang H. A Novel Bayes Model: Hidden Naive Bayes. IEEE Trans. Knowl. Data Eng. 2009; 21(10): 1361-1371. http://dx.doi.org /10.1109/TKDE. 2008.234

[17] Sorensen L, Nielsen M, Lo P. Texture-based analysis of COPD: a data-driven approach. IEEE Trans. Med. Imaging. 2012; 31(1): 70-78. 